\title{
Hepatobiliary Fascioliasis Presenting as Choledocholithiasis: A Case Report
}

\author{
Terence 0’Keeffe* \\ Department of Surgery, University of Arizona, USA
}

*Corresponding author: Terence O'Keeffe, MBChB, FACS, MSPH, Department of Surgery, Division of Trauma, Critical Care, Burns and Emergency Surgery, University of Arizona, 1501 N. Campbell Ave, Room.5411, P.O. Box 245063, Tucson, AZ 85727, USA

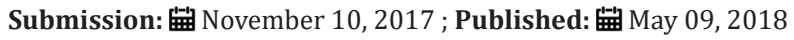

Keywords: Liver flukes; Fasciola hepatica; Biliary obstruction; Endoscopic retrograde cholangiopancreatography; Cholelithiasis; Cholecystectomy

\section{Case Report}

A 28 year-old Hispanic female who was 36 weeks pregnant presented to our hospital complaining of 2 days of intermittent right upper quadrant abdominal pain, nausea, and vomiting. She denied any fever, chills, jaundice, changes in her bowel pattern, or recent travel, and had no significant past medical or surgical history. Physical examination revealed a gravid abdomen with mild right upper quadrant tenderness on palpation. Liver function tests (LFTs) were abnormal on admission with an aspartate amino transferase level (AST) of 76 International Units/Liter (IU/L), alanine amino transferase (ALT) of 61IU/L, alkaline phosphatase (AlkPhos) of 226IU/L, total bilirubin (TB) of 1.9 milligrams/deciliter (mg/dL), and a direct bilirubin (DB) of $0.6 \mathrm{mg} / \mathrm{dl}$. Abdominal ultrasound revealed cholelithiasis with a common bile duct diameter of nine millimeters. She was admitted to the antepartum unit with resolution of her symptoms and normalization of her LFTs within forty-eight hours. Due to the patient's advanced pregnancy, outpatient elective laparoscopic cholecystectomy was scheduled for the postpartum period and she was discharged.

One week post-partum, the patient returned to the emergency department with recurrent abdominal pain and vomiting. She appeared icteric with right upper quadrant abdominal tenderness to palpation. Laboratory values included a white blood cell count of $6,400 / \mu \mathrm{l}$, AST of 117IU/L, ALT of 182IU/L, AlkPhos of 358IU/L, TB of $3.7 \mathrm{mg} / \mathrm{dl}$, and a DB of $1.8 \mathrm{mg} / \mathrm{dl}$. Amylase and lipase levels were normal. A repeat abdominal ultrasound showed cholelithiasis with a common bile duct measuring three millimeters without evidence of acute cholecystitis. The patient was admitted to the surgical service with a presumptive diagnosis of choledocholithiasis and started on intravenous antibiotics. Her hyperbilirubinemia worsened with her TB increasing to $4.5 \mathrm{mg} / \mathrm{dl}$ within twelve hours of presentation, prompting Endoscopic Retrograde Cholangiopancreatography (ERCP) for suspected biliary tract obstruction.

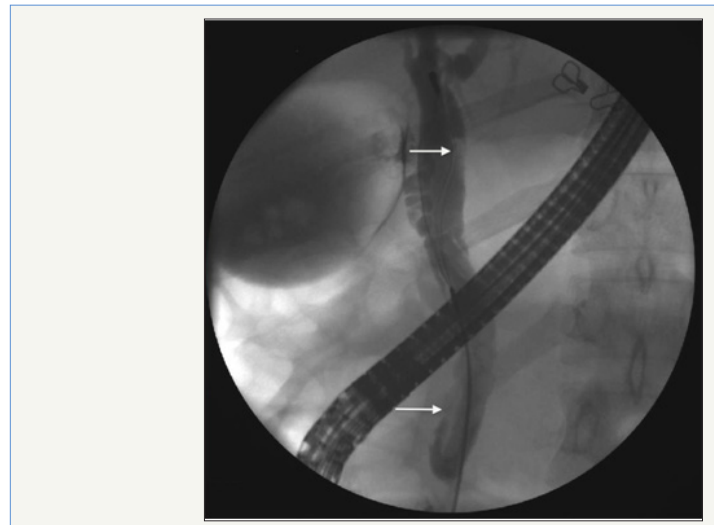

Figure 1: ERCP demonstrating multiple filling defects.

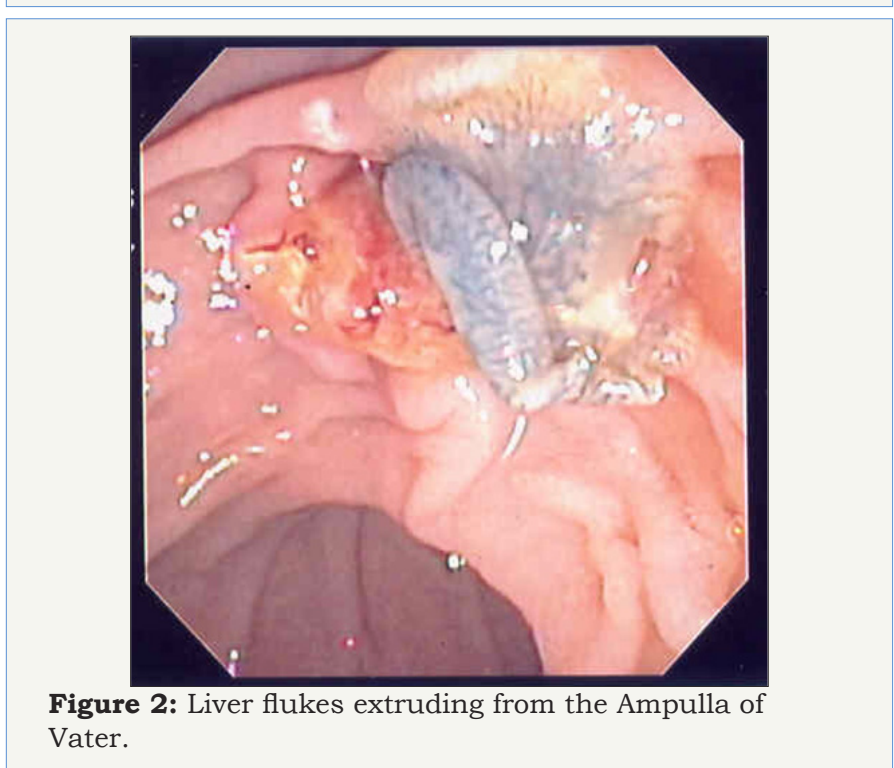

This revealed a normal esophagus, stomach, and duodenum. The ampulla was cannulated without difficulty and the initial 
cholangiogram showed an extrahepatic bile duct of $12 \mathrm{~mm}$ in diameter with several small filling defects (Figure 1). After performing a generous sphincterotomy, we were surprised to encounter three live liver flukes, which were extracted from the distal common bile duct (Figures $2 \& 3$ ). Follow-up cholangiography revealed no filling defects. Stool studies for ova and parasites were collected and positive for Fasciola hepatica. An enzyme-linked immunoabsorbent assay (ELISA) test was also positive for Fasciola hepatica, with a titer of $1: 16$. The recommendation by our infectious disease specialists was for treatment with Triclabendazole $750 \mathrm{mg}$ orally for two days. Following relief of her biliary duct obstruction, the patient improved clinically and was discharged from the hospital. As Triclabendazole is not readily available in the United States, it was sourced from Switzerland. The patient completed the therapy as an outpatient and ova and parasite stool studies were negative after treatment. A repeat abdominal ultrasound showed a contracted gallbladder with persistent echogenic material in the lumen and a common bile duct diameter of 2.5 to $4.2 \mathrm{~mm}$. The patient underwent a successful outpatient laparoscopic cholecystectomy three months after her discharge. The specimen was examined in the operating room and found to only contain small gallstones. Pathologic examination of the gallbladder revealed chronic cholecystitis and cholesterolosis.

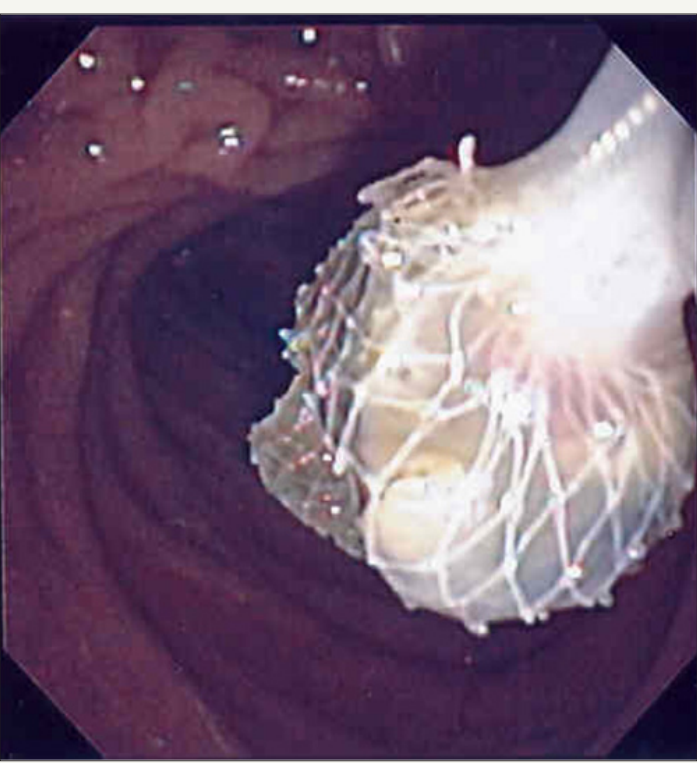

Figure 3: Liver flukes being extracted using a endoscopic basket.

We initially suspected our patient had cholelithiasis with choledocholithiasis in light of the previous biochemical abnormalities during her initial presentation, and her known cholelithiasis. We elected to perform preoperative ERCP given her worsening hyperbilirubinemia and concern for possible cholangitis. ERCP was instrumental in obtaining the correct diagnosis and facilitating appropriate treatment. Proceeding directly to cholecystectomy would not have addressed the biliary obstruction from the adult flukes, and would have likely been extremely challenging because of this parasitic infection.
There is a paucity of English-language literature regarding chronic Fasciola hepatica infection as an etiology of obstructive jaundice, most likely due to the rarity of this clinical entity. Infection is most common in endemic areas, which include Europe, Latin America, Australia, Asia, and the Middle East. Transmission to humans occurs from consumption of freshwater plants (e.g. watercress) that contain cystic forms of the fluke, or by drinking contaminated water. The organism subsequently migrates through the wall of the proximal gastrointestinal tract to enter the peritoneal cavity, where it penetrates the Glisson capsule and invades the hepatic parenchyma [1].

Clinical manifestations of fascioliasis typically occur in two distinct phases. Acute infection represents invasion of the hepatic parenchyma by the fluke. Classic findings include fever; right upper quadrant pain, and hepatomegaly. Eosinophilia is the most common laboratory finding during this phase of infection. Mild elevations in liver function tests such as AST, ALT, and AlkPhos may also be present. ELISA has a sensitivity of approximately 98\% for the detection of excretory-secretory antigen products from the adult fluke during acute infection. Ova and parasite stool studies are negative in this phase. Complications of acute fascioliasis include ascites, hepatitis, subcapsular hemorrhage, and hepatic necrosis. Migration of the organism into the lungs, pleura, pericardium, abdominal wall, and brain has been reported. While approximately $18 \%$ of acute infection with Fasciola hepatica is asymptomatic, the manifestations may last up to three months.

Chronic infection with Fasciola hepatica results from migration of the fluke from the liver into the biliary tree. This can result in chronic inflammation of the extrahepatic biliary tree with hyperplasia of the ductal epithelium and fibrosis. Most chronic cases remain asymptomatic. Manifestations may include recurrent right upper quadrant pain, episodes of jaundice, and fluctuating liver enzymes. Diagnosis in this phase requires a high index of suspicion. No serological markers are useful, as eosinophilia and positive ELISA studies are uncommon with chronic infection. Duodenal fluid aspirates or stool studies may detect parasite ova. Complications of chronic fascioliasis include cholangitis, pancreatitis, and obstructive jaundice. Chronic Fasciola hepatic infection has not been associated with hepatobiliary malignancy.

Radiologic studies may aid in increasing clinical suspicion of hepatobiliaryfascioliasis. In a case series by Sezgin et al, all nine patients with fascioliasis had dilatation of the intrahepatic and extrahepatic bile ducts on abdominal ultrasound [2]. Over fifty percent had thickened bile ducts associated with hypoechoic peripheral nodular lesions in the liver. Flukes may also be visualized in the gallbladder as hypoechoic structures lacking acoustic shadowing typical of cholelithiasis. Computed Tomography (CT) scanning may demonstrate multiple low density, contrasting enhancing nodules within the hepatic parenchyma representing microabscesses and necrosis from invasion of the organism. Nodules associated with linear tracts are highly suggestive of fascioliasis. Ductal dilation, hepatomegaly, splenomegaly, regional 
lymphadenopathy, and flukes within the gallbladder may also be seen on CT scan.

Endoscopic retrograde cholangiopancreatography (ERCP) is a valuable tool in both the diagnosis and treatment of chronic fascioliasis [3-5]. Linear or crescent-shaped filling defects on cholangiography are suggestive of adult organisms within the bile ducts. Direct visualization and extraction of the flukes is often possible, as in our case. Clearance of the bile ducts usually results in symptomatic improvement. In addition to ERCP, treatment with antiparasitic medications is also recommended. Multiple drug regiments have been used, including mebendazole, albendazole, and praziquantel. Current recommendations are for therapy with either triclabendazole or bithionol, both of which may be difficult to obtain in the United States. Indications for cholecystectomy in patients with biliary fascioliasis have not been well delineated, although there is a case report of a living fluke encountered at the time of cholecystectomy [6]. We are not aware of any reports of chronic fascioliasis with concurrent cholelithiasis in the literature.

Fasciola hepatica is an extremely rare cause of biliary tract obstruction in the United States. It is a challenging diagnosis to make but in view of increasing immigration of patients from endemic regions, a high index of suspicion should be maintained in patients with atypical presentations of choledocholithiasis. The treatment of choice should be anti-parasitic medication, followed by cholecystectomy in these cases, with ERCP if necessary to provide clearance of the extra-hepatic bile ducts from adult flukes. An awareness of this rare disease is beneficial in providing appropriate care.

\section{Funding}

There were no sources of funding or conflicts of interest in this study.

\section{References}

1. Atalay F, Kirimlioglu V, Dagli U (1993) Human fascioliasis. Surg Today 23: 366-369.

2. Sezgin O, Altintas E, Disibeyaz S, Saritaş U, Sahin B (2004) Hepatobiliary fascioliasis: clinical and radiologic features and endoscopic management. J Clin Gastroenterol 38(3): 285-291.

3. Danilewitz M, Kotfila R, Jensen P (1996) Endoscopic diagnosis and management of Fasciola hepatica causing biliary obstruction. Am J Gastroenterol 91(12): 2620-2621.

4. Fullerton JK, Vitale M, Vitale GC (2006) Therapeutic endoscopic retrograde cholangiopancreatography for the treatment of Fasciola hepatica presenting as biliary obstruction. Surg Innov 13(3): 179-182.

5. Gulsen MT, Savas MC, Koruk M, Kadayifci A, Demirci F (2006) Fascioliasis: a report of five cases presenting with common bile duct obstruction. Neth J Med 64(1): 17-19.

6. Bulbuloglu E, Yuksel M, Bakaris S, Celik M, Kokoglu OF, et al. (2007) Diagnosis of fasciola hepatica cases in an operating room. Trop Doct 37(1): 50-52.
Creative Commons Attribution 4.0 International License

For possible submissions Click Here

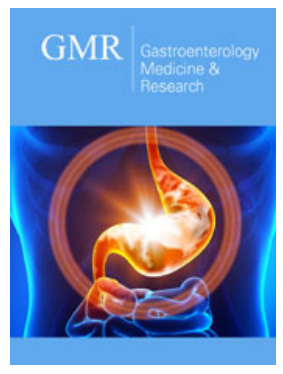

Gastroenterology Medicine \& Research

\section{Benefits of Publishing with us}

- High-level peer review and editorial services

- Freely accessible online immediately upon publication

- Authors retain the copyright to their work

- Licensing it under a Creative Commons license

- Visibility through different online platforms 